\title{
Design and Development of Smart Fleet System using Global Positioning System
}

\author{
Swapna P, A. Daisy Rani
}

\begin{abstract}
Now a days, Vehicle tracking system plays a major role in our daily life. As the technology grows, vehicle thefts are increasing enormously. This paper proposes to design an embedded system which is used to track and position any vehicle by using Global Positioning System (GPS) and Global system for mobile communication (GSM). This helps in monitoring and reporting the status of the moving Vehicle on demand continuously. So, ATMEGA328 microcontroller is serially interfaced to a GSM Modem and GPS Receiver through serial communication protocol RS 232. In this, driver circuit is used to covert TTL voltages into RS 232 voltage levels. Identifying the position of the remote vehicle is done by GPS modem continuously. The current location details like vehicle longitudes and latitudes of the remote vehicle is sent through GSM modem. The output is acquired from GPS modem and displayed on the LCD display. The same data is transmitted to the mobile at the other end from where the position of the vehicle is demanded. Based on the request placed by the user, the position of the vehicle is automatically sent to the corresponding mobile number. So, this project has been implemented to identify lost vehicle, know the status of moving vehicle from remote location and send the information to the user's mobile number.
\end{abstract}

Keywords: Microcontroller, GPS, GSM, Vehicle tracking system, Embedded systems.

\section{INTRODUCTION}

Navigation of vehicles is one of the important applications in the field of navigation which is primarily used by drivers. The digital maps given to the driver play an important role in this field. When larger objects or vehicles were spread out on the roads, the owners often found it difficult to track and be aware of what was happening with their vehicle. Vehicle tracking system gives the information regarding location of the vehicle, distance travelled at a specific time. Also the need of tracking vehicle is to prevent any kind of theft.E.D Kalpan et al has discussed about GPS based tracking system for public transport system such as buses, taxis etc. In this paper, On-board module consists of GSM modem and ARM processor. The navigation message was broadcasted through GPS and a message was sent through GSM communication[1]. Chia-Hung Lein et al have developed a FPGA based vehicle tracking and accident warning system. The location of the vehicle was identified by GPS and an accelerometer sensor was used to detect any kind of accidents to the vehicle[2].

Revised Manuscript Received on February 05, 2020.

* Correspondence Author

Dr Swapna, Assistant Professor, Dept. Of Instrument Technology, AUCE(A) Visakhapatnam, Andhra Pradesh, India. Email: peravali_r2000@yahoo.co.in

Dr A. Daisy Rani, Assistant Professor, Dept. Of Instrument Technology, AUCE(A) Visakhapatnam, Andhra Pradesh, India. a_daisrani@gmail.com

(c) The Authors. Published by Blue Eyes Intelligence Engineering and Sciences Publication (BEIESP). This is an open access article under the CC BY-NC-ND license (http://creativecommons.org/licenses/by-nc-nd/4.0/)
A. Goel at al has implemented advanced vehicle tracking system on Google earth using GPS and GSM for commercial vehicles to get the information of vehicle's location and distance for specific time intervals defined by the user. Then this information is transmitted to monitoring or tracking server. This transmitted information is displayed on the display unit using Google earth to display vehicle location[3]. Some of the researchers have developed GPS and GSM based vehicle tracking system with the help of PIC microcontrollers[4][5][6]. In this paper, section 1 deals with importance of vehicle tracking system and literature survey have presented on present research trends on GPS based vehicle tracking systems. Section 2 deals with introduction to hardware and specifications of each component. Section 3 deals with block diagram and methodology used in this work. GPS and GSM based vehicle tracking system using microcontroller results on various conditions are discussed in section 4. Finally, the conclusion and future scope of the present work is carried out in this paper.

\section{HARDWARE MODULES}

1. Embedded systems: An embedded system can be defined as a computing device that does a specified job. Examples naming air-conditioner, VCD player, DVD player, printer, fax machine, mobile phone etc. All the appliances consist of a processor and additional hardware as per the requirement based on application. It has very limited resources, particularly the memory and need to work against some stringent deadlines. Missing a deadline may cause a catastrophic loss to life or property. Every embedded system consists of built-in Central Processing Unit (CPU). The hardware also contains memory chips and software usually loaded into it.

2. Arduino Microcontroller: Microcontroller is a single chip computer embedded on small circuit board, that is used to perform calculations, tasks and send/receive signals from other devices. Arduino Uno is one such microcontroller board. It is an open source electronics prototyping platform. The actual microcontroller at its hear is the chip called AtMega328. The advantages are largely in terms of reliability of the circuit hardware as well as the ease of programming. this work is carried out in Arduino Uno hardware board (Figure 2.1) with Arduino IDE software.

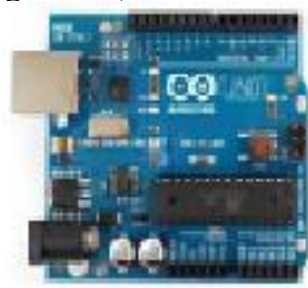

Figure 2.1: Arduino Board 
3. GSM Module: GSM (Global System for Mobile communication) is an open, digital cellular technology used to transmit data services. It is a digital telephone system which is used in various parts of the world. GSM uses a variation of Time Division Multiple Access (TDMA). GSM networks operate in different frequency ranges. Most 2G GSM networks operate in 900MHz. GSM-900 uses 890-915 $\mathrm{MHz}$ to send information from the mobile station to the base station (uplink) and $935-960 \mathrm{MHz}$ for the other direction(downlink), providing $124 \mathrm{RF}$ channels speed at 200KHz. GSM modem cannot be directly connected to a microcontroller. So, RS 232 serial communication is used to interface GS modem with the microcontroller as shown in figure2.2.

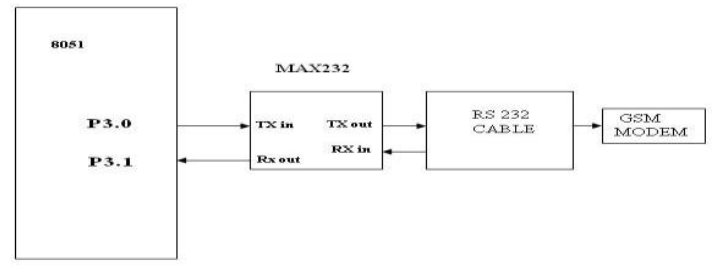

Figure 2.2: Serial communication through Max 232

4. GPS Receiver with antenna: GPS receivers provide the three dimensional location (Latitude, longitude and altitude) plus precise time which is broadcasted by the Global positioning system satellites from space. GPS receivers provide reliable position, navigation and timing service to the world on a continuous basis in all weather conditions, day and night and anywhere on or near the Earth. The features are high sensitivity - $160 \mathrm{dBm}$, searching upto 65 channels of satellites.

The block diagram as shown in Figure 3.1 tells about how the interfacing of various modules are done and connections established with microcontrollers. The microcontroller is the heart of the block diagram and program in it is the key for functioning. It consists of GPS, GSM, trespass sensor, fire sensor, Max 232 and LCD display.

Switch on $12 \mathrm{~V}$ step-up transformer power supply and make every component in active condition. Aurdino Uno R3 is the controller used in it. After switch on the power supply, Microcontroller is ready to receive the signal.

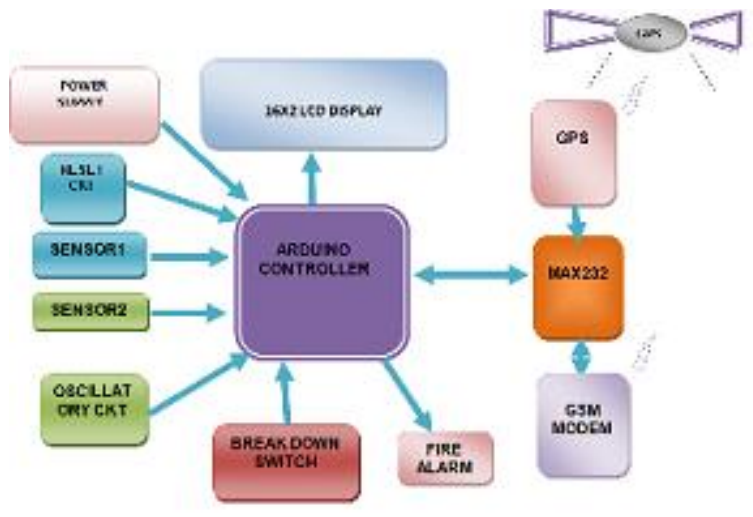

Figure 3.1: Bock Diagram

Initially, GPS module receives the signals from satellite. This information is given to the microcontroller and then displayed on LCD display. Now, LCD displays the GPS values such as latitude and longitude (position of the current vehicle). In the Figure sensor 1 is IR sensor used to count the number passengers on board and display its value on LCD display board. GSM module receive the information from the microcontroller and send the messages on demand like vehicle breakdown, fire alert and location of vehicle to the user. Sensor 2 in the figure is IR fire sensor to detect flame present in the vehicle and send the message to particular contact number given in the program. Fire alarm is activated if any fire occurs in the vehicle. Max 232 is allowed for data transfer serially from microcontroller to RS 232. The information collected though Max 232 is passed on to GSM and GPS module vice-versa. The data from all the modules are collected in parallel to the microcontroller and displayed on LCD display board. The implemented project is shown in figure 3.2 .

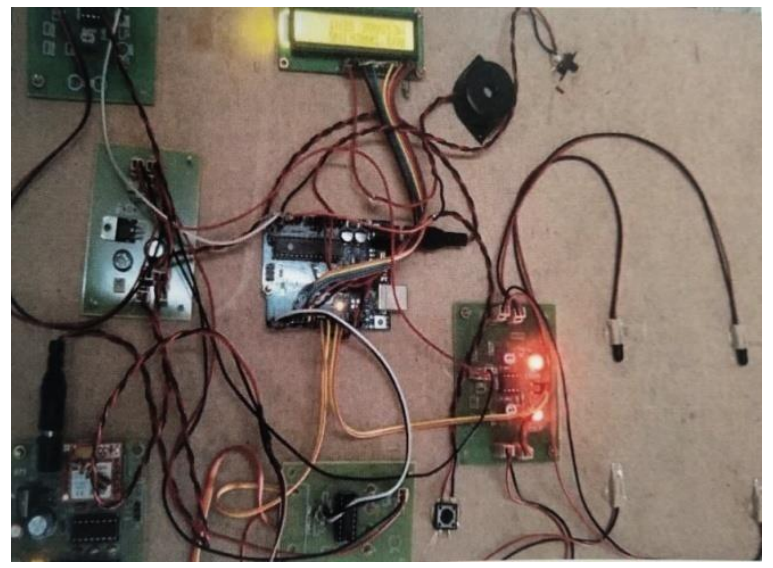

Figure 3.2: Circuit developed in this work

\section{RESULTS AND DISCUSSION}

In this, Arduino IDE software is programmed to track the current location of the vehicle. The module is simulated under various conditions and sent the SMS to the contact number through GSM protocol. The same information is displayed on the LCD display.

Case 1: In figure 4.1, LCD display is displaying a message as the vehicle is under monitoring immediately after connecting to the vehicle.

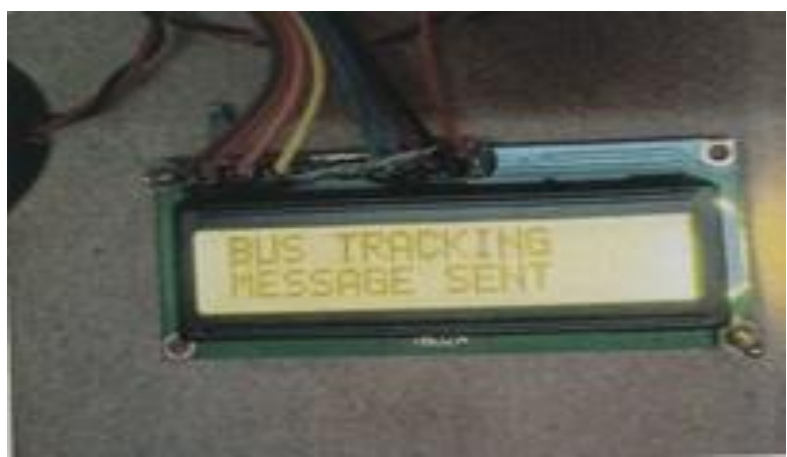

Figure 4.1: Bus tracking message displayed on LCD

Case 2: If the vehicle breaks down, then LCD display shows that tracking vehicle breakdown (Figure 4.2).

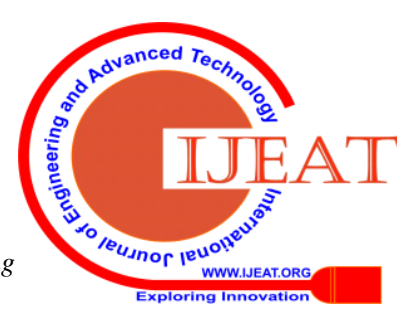




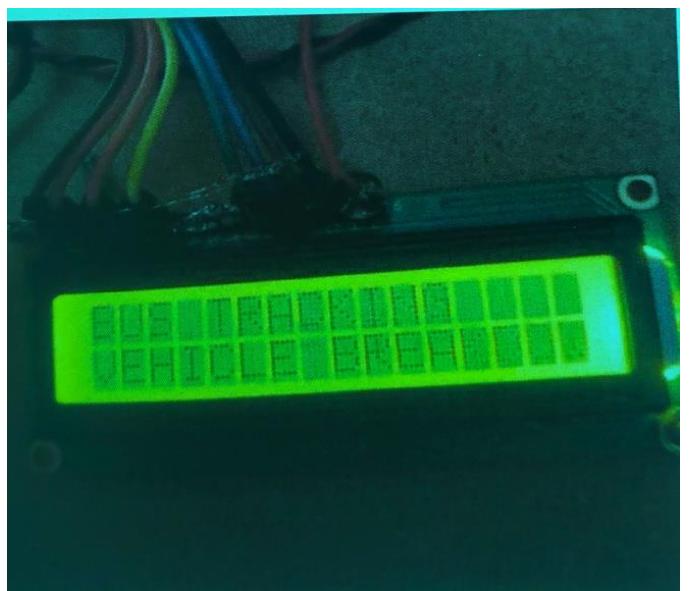

Figure 4.2: vehicle breakdown message displayed

Case 3: If the fire is detected in the vehicle through fire sensor, then LCD display shows that tracking vehicle fire detected (Figure 4.3).

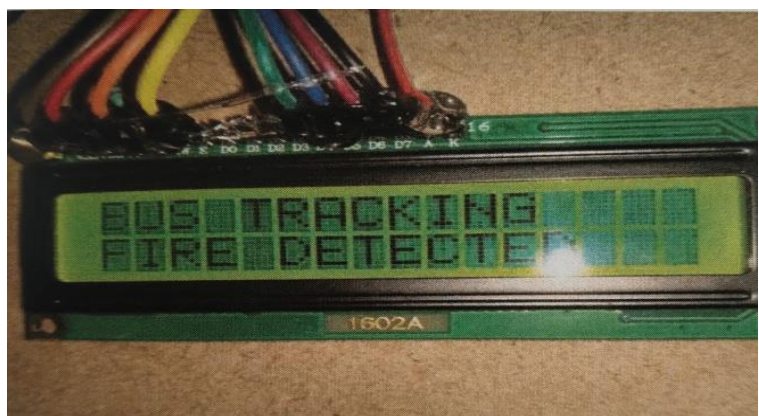

Figure 4.3: Fire detection message displayed on LCD

case 4: Based on the user request, it gives information about the number of passengers on board, location of the vehicle in google map and alarm is given of any fire detection or breakdown of the vehicle(Figure 4.4, 4.5).

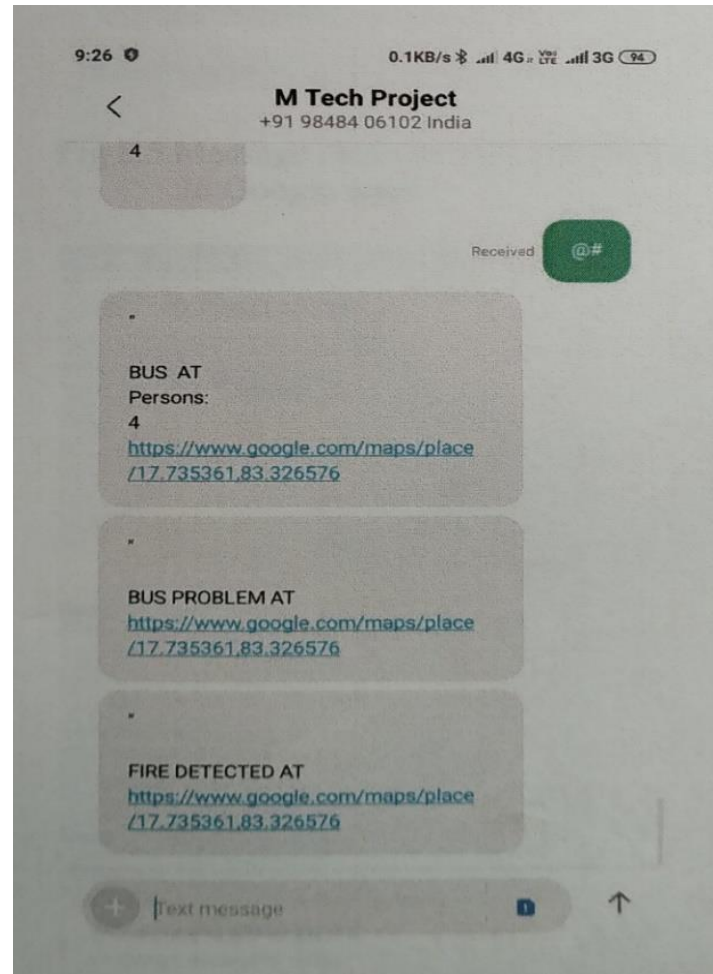

Figure 4.4: Display the number persons on board information received to mobile phone.

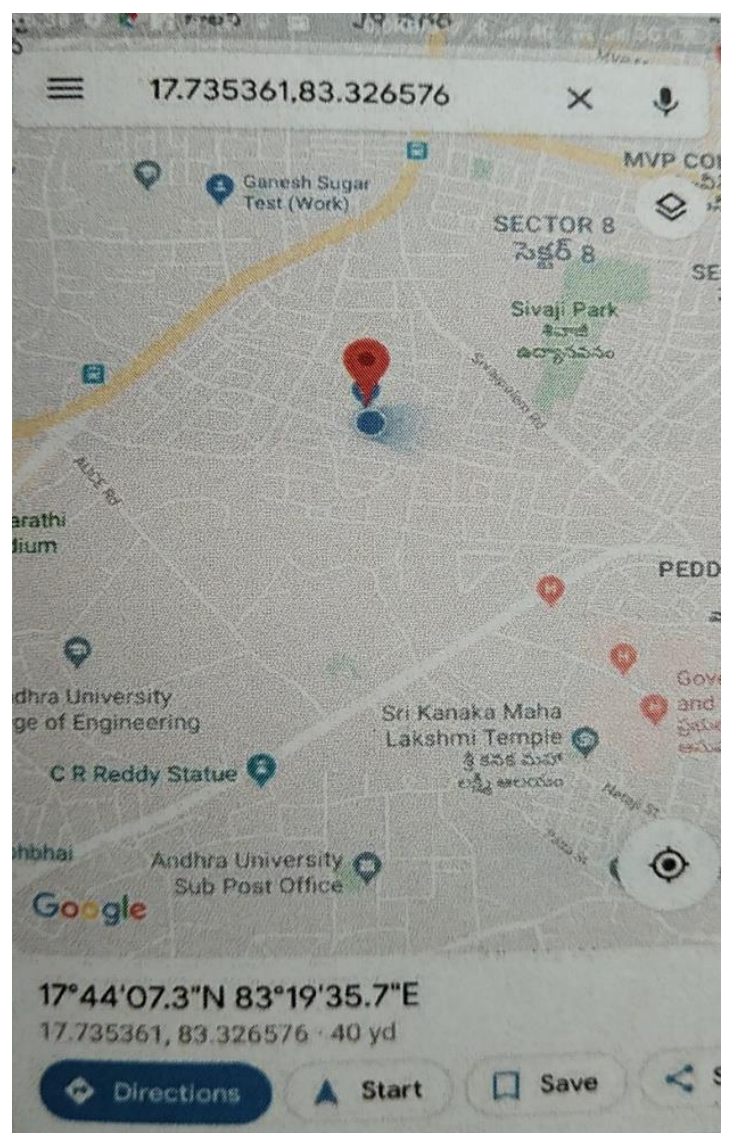

Figure 4.5: Locating vehicle in Google map

In all the cases, the information is send as SMS to the concern owner/person contact number. So this project helps in anti-theft, avoid fire accidents and give alarm when in emergency.

\section{CONCLUSION}

By installing this circuit in any vehicle allows us to track vehicles like public vehicles and private vehicles. The hardware designed is portable and easy to install in any vehicle. In this, GSM and GPS modems are used to track the vehicle and send information to the concerned person. The information related to the vehicle like position based on longitudes and latitudes are captured by GPS module. Microcontroller is used to receive the information from GPS and send the same to the owner of the vehicle through GSM. Apart from this, fire detection and number of passengers onboard is collected by an IR sensor. If fire is detected in the vehicle, microcontroller gives an alarm. So, this project is very much useful in real time scenario.

\section{REFERENCES}

1. E.D. Kalpan, "Understanding GPS: Principles and Applications", Artech Huse publishers, ISBN 0890067937, February 1996.

2. Chia-Hung Lein, Chi-Hsiung Lin, Ying-Wen Bai, Ming-Fong Liu and Ming-Bo Lin " Remotely controllable outlet system for home power management" Proceedings of IEEE tenth international symposium on consumer electrnics (ISCE 2006), St Petersburg, Russia, PP-7-12, 2006.

3. A Goel and V. Gruhn " Fleet monitoring system for Advanced tracking of commercial vehicles", Proceedings of IEEE International conference on systems, man and cybernetics (SME 2006), PP 25172522, Taipei, Taiwan, 2006.

Published By: 
4. Junaid Ali, Shaib Nasim, Taha Ali, Naveed Ahmed and Syed Riaz un Nabi "Implementation of GSM based commercial automobile tracker using PIC18F452 ad development of Google Earth Embedded monitoring software" Proceedings of IEEE conference on research and development (SCOReD 2009), PP 16-19, UPM Serdang, Malaysia.

5. M. Mc Donald, H. Keller, J. Klijnhout and V. mauro, " Intelligent Transport system in Europe: Opportunity for future research" World scientific publishing company, ISBN 981270082, 2006.

6. Muhammad Ali Mazidi, Janice Gillspie, M Kinlay, Rolin D "The microcontroller in Embedded system using Assembly and C", 2nd edition published by Pearson Education, 2009.

\section{AUTHORS PROFILE}

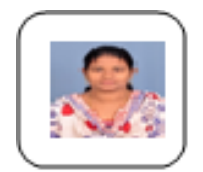

Dr Swapna $\mathbf{P}$ received her graduation degree(B.Tech) in Electronics and Instrumentation Engineering from Acharya Nagarjuna University, Guntur, Andhra pradesh in 2000, Masters degree (M.Tech) from National Institute of Technology (NIT) Calicut, Kerala in 2002 and Ph.D degree from department of Instrument Technology, Andhra University, Andhra pradesh in 2015. Her areas of research are modern control systems, predictive control systems, microcontroller based intelligence systems and Solar PV systems. She has published more than 15 journals in various National and International journals in her research areas. Presently working as assistant professor in the department of Instrument Technology, AU College of Engineering(A), Andhra University, Visakhapatnam, Andhra Pradesh.

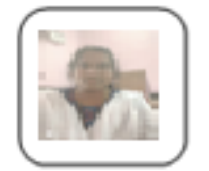

Dr A Daisy Rani received a Ph.D degree in MEMS Technology and Masters degree in Electronics and Instrumentation Engineering from college of Engineering, Andhra University (AU) in 2015 and 2004 respectively. She is working as assistant professor in the department of Instrument Technology in Andhra University. The MEMS design, NANO Technology and low power VLSI digital circuits are her area's of research. She published more than 18 International journals in her area of interest 